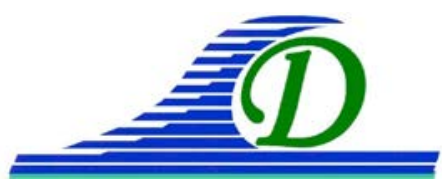
XIII ${ }^{\text {èmes }}$ Journées Nationales Génie Côtier - Génie Civil Dunkerque, 2-4 juillet 2014

DOI:10.5150/jngcgc.2014.090 @ Editions Paralia CFL

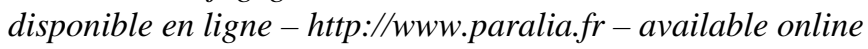

\title{
Un SIG pour appuyer la gestion durable du littoral : exemple de l'érosion en Pays de Monts (Vendée, France)
}

\author{
Cécile LE GUERN ${ }^{1}$, Vivien BAUdOUIN ${ }^{1}$, Marc ROBIN ${ }^{2}$, Paul FATTAL $^{2}$, \\ Pierre CONIL ${ }^{1}$, Martin JUIGNER ${ }^{2}$, Françoise DEBAINE ${ }^{2}$, \\ Mohamed MAANAN ${ }^{2}$, Christophe ROLLIER ${ }^{3}$, Loïc GOUGUET ${ }^{3}$, \\ Frédéric BOUCHET ${ }^{4}$, Jean MAGNE ${ }^{4}$, Jean-Guy ROBIN ${ }^{4}$
}

1. BRGM, Direction Régionale des Pays de la Loire, 1 rue des Saumonières, BP 92342, 44323 Nantes, France.c.leguern@brgm.fr

2. Institut de Géographie et d'Aménagement Régional, IGARUN/GéolittomerUMR6554, LETG CNRS, 44312 Nantes Cedex, France.Paul.Fattal@univ-nantes.fr

3. ONF, DT Centre-Ouest Auvergne Limousin, 15 Boulevard Léon Bureau - CS 13 237, 44262 Nantes Cedex 2, France. christophe.rollier@onf.fr

4. Communauté de Communes Océan-Marais-de-Monts, Communauté de Communes Océan-Marais de Monts, 46, place de la Paix, BP 721, 85167 Saint Jean de Monts, France.frederic.bouchet@omdm.fr

\section{Résumé :}

Décidé en 2009 par la Communauté de Communes Océan Marais de Monts, l'Observatoire du Littoral du Pays de Monts, vise par son caractère pérenne à constituer un véritable outil d'aide à la gestion prévisionnelle du trait de côte. Il couvre un linéaire côtier sableux de $19 \mathrm{~km}$ allant de La-Barre-de-Monts (Fromentine) à Saint-Jean-deMonts (plage des Demoiselles) en Vendée.

Le cœur de l'observatoire est un système d'informations géographiques (SIG), qui intègre les données pertinentes issues d'un point sur les connaissances existantes, ainsi que les suivis de terrain (état initial, suivis réguliers et événementiels). Son intérêt pour appuyer la gestion durable du territoire est illustré à partir de l'exemple de l'érosion du trait de côte, avec la comparaison court terme (volume de sable exporté par Xynthia)/long terme (évolution du trait de côte sur 100 ans).

Le bilan de la mise en place de l'observatoire, au bout de 5 ans, est (a) un SIG en cours de finalisation avant livraison à la collectivité, (b) des interprétations des observations et phénomènes en jeu et (c) des premières réponses aux questions de la collectivité ainsi que des premières mises en application de solutions (fils lisses, ...).

Mots-clés : GIZC, Observatoire, Littoral, Trait de côte, Sable, Dune, Base de données, SIG, Erosion, Ecosystèmes côtiers, Aide à la décision, Pays de Monts, Vendée, France.

\section{Introduction}

Dans un contexte général d'érosion littorale (1/4 côtes françaises métropolitaines, IFEN, 2007), en corrélation avec l'attractivité toujours plus importante des zones côtières, les 


\section{Thème 6 - Gestion durable des zones littorales et estuariennes}

collectivités doivent prendre en compte le risque dans leur politique de développement territorial. Faute d'une vision suffisamment globale du littoral et d'une connaissance suffisante des phénomènes, les solutions de gestion (épis, enrochement, ...) mises en œuvre dans le passé et encore récemment, reportent souvent les problèmes d'érosion sur le territoire voisin.

Cette problématique montre tout l'intérêt du concept assez récent de Gestion Intégrée des Zones Côtières (GIZC), qui consiste à rechercher un certain équilibre dans les priorités d'action, en adoptant une approche de l'espace plus dynamique et intégrée. Les démarches d'observation du littoral y jouent un rôle central (BULTEAU et al., 2011). Elles s’inscrivent pleinement dans la "Stratégie nationale de gestion intégrée du trait de côte" élaborée en 2012 par le Ministère de l'écologie, du développement durable et de l'énergie. Cette stratégie fait suite au grenelle de l'environnement, la tempête Xynthia (2010) ayant par ailleurs marqué les esprits et renforcé la prise de conscience sur l'intérêt des démarches d'observation.

Face à un constat de recul d'une partie de son trait de côte et au manque de données existantes sur sa frange littorale, la Communauté de Communes Océan-Marais de Monts (Vendée, France) a décidé en 2009 de créer un "Observatoire du littoral". Cette structure, par son caractère pérenne, a pour objectif de constituer un véritable outil d'aide à la gestion prévisionnelle du trait de côte. Le présent article présente les principales actions menées dans le cadre des 5 premières années de vie de l'observatoire, avec un focus sur l'utilisation du SIG en cours d'élaboration. Dans ce cadre, la comparaison de l'érosion côtière sur le court et le long terme est prise comme exemple.

\section{Le littoral des Pays de Monts}

L’observatoire du littoral du Pays de Monts (Vendée, France) couvre un linéaire côtier sableux de 19 km allant de Fromentine (La-Barre-de-Monts) à la plage des Demoiselles (Saint-Jean-de-Monts) (Figure 1). Ce littoral est caractérisé par un cordon dunaire important, composé de sables récents et actuels. Il est marqué en son milieu par le Pont d'Yeu, secteur présentant un substratum rocheux subaffleurant, qui correspond à des calcaires éocènes présents également au niveau de platiers rocheux, et en sousbassement de la zone rétro-littorale (THINON et al., 2013). Cette dernière, constituée de terrains argileux d'origine marine (transgression flandrienne), est connue sous le nom de Marais de Monts.

Les dunes non-boisées représentent environ 12\% de la surface du massif dunaire des Pays de Monts (PRAT, 2008). L'espace dunaire, d'environ $10 \mathrm{~m}$ d'altitude moyenne sur sa façade maritime, atteint plus de 20 mètres sur certains cordons. En partie fixé par reboisement au cours du XIX siècle, il comprend plusieurs cordons dunaires issus d'accrétions successives à la fin de l'Holocène et est caractérisé par des dunes de type parabolique bien visibles sur le levé Lidar Litto3D seuillé (figure 1). 


\section{XIII ${ }^{\text {èmes }}$ Journées Nationales Génie Côtier - Génie Civil \\ Dunkerque, 2-4 juillet 2014}

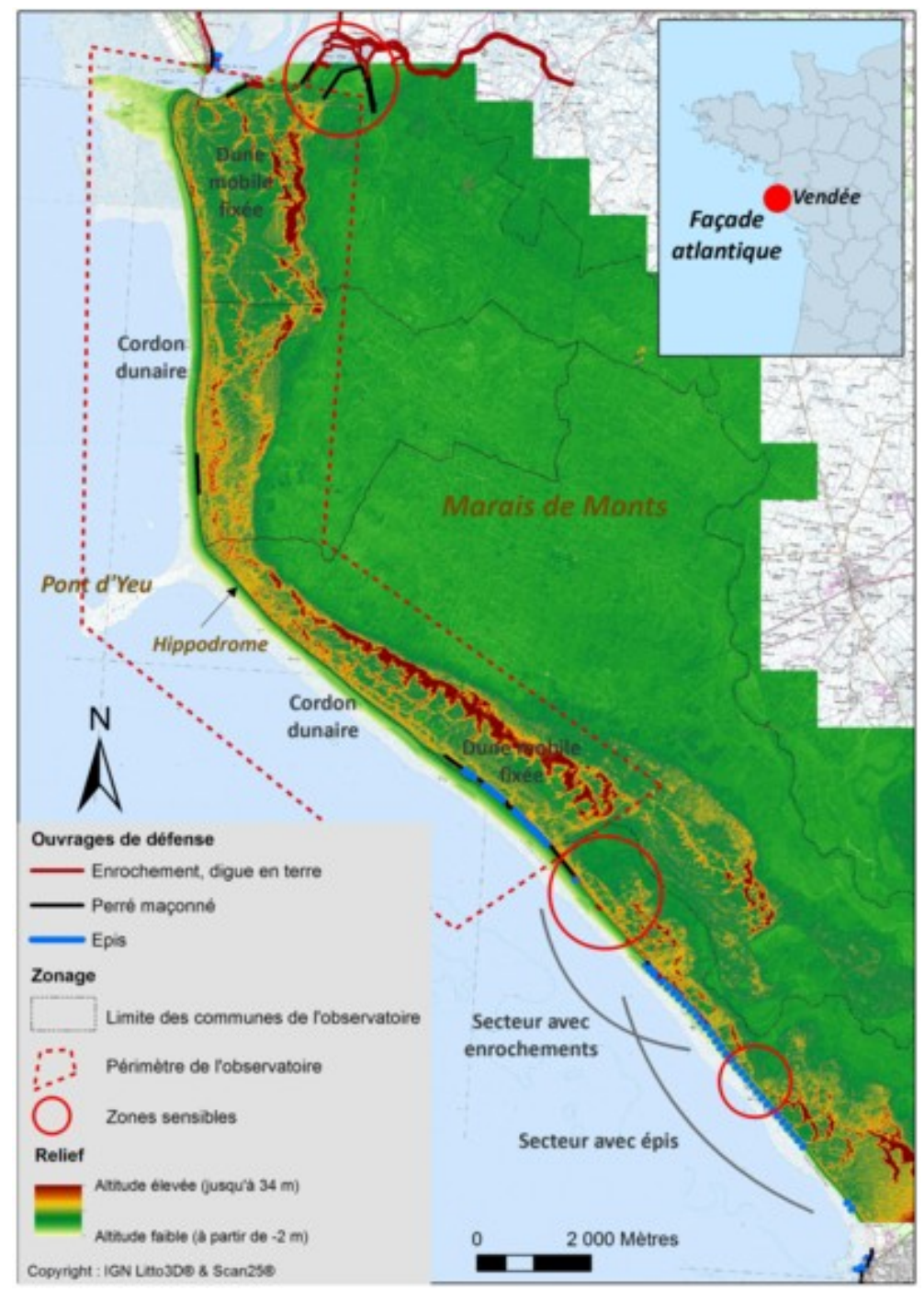

Figure 1 : Topographie du territoire du Pays de Monts

Les enjeux sur cette portion de littoral sont multiples et antagonistes, avec des activités économiques à maintenir et un milieu naturel et sa biodiversité à protéger (dunes intégrées au réseau Natura 2000). Il importe ainsi de prendre en compte ces enjeux en plus des forçages météo-marins dans la gestion du littoral, qui représente un système complexe multidimensionnel. Ainsi par exemple, si les activités touristiques profitent de l'environnement préservé (plages, dunes) du littoral du Pays de Monts, la fréquentation touristique importante en été (près de 13000 habitants sur le territoire de la communauté de commune en hiver, près de 10 fois plus en été) y crée une pression importante, pouvant favoriser l'érosion et la perte de biodiversité.

Pour le moment la pression érosive ne met pas la Communauté de Communes des Pays de Monts en situation d'urgence, contrairement à d'autres portions du littoral vendéen et charentais que la tempête Xynthia a mises en évidence. Toutefois, consciente des évolutions du climat (élévation du niveau des mers et augmentation attendue de la 


\section{Thème 6 - Gestion durable des zones littorales et estuariennes}

fréquence et de la force des tempêtes), et inquiète des reculs de trait de côte constatés, la collectivité préfère anticiper et privilégier les solutions douces de gestion.

\section{Le SIG, outil de gestion des données et d'aide à la décision}

Créé en 2010 et intégré au SOERE "Trait de côte" en 2012 puis au SNO (Service National d'Observation) sur le trait de côté labellisé par l'INSU (Institut National des Sciences de l'Univers, CNRS) en 2014, l'observatoire du littoral des Pays de Monts s'appuie sur une étroite collaboration entre la collectivité et plusieurs partenaires scientifiques et techniques : Université de Nantes (LETG-IGARUN), ONF, BRGM.

Pour mettre en place et faire vivre l'observatoire dans le temps, un ensemble d'actions a été engagé. Elles s'appuient notamment sur l'expérience commune de l'ONF et du BRGM dans l'observatoire de la Côte Aquitaine (MUGICA et al., 2009 ; MALLET et al., 2013 ; http://littoral.aquitaine.fr), et sur l'expérience de gestion du littoral de l'IGARUN (travaux d'étudiants en masters, thèses et projets de recherche). L'état des lieux, la structuration et la mise en forme des données ainsi que la compréhension des phénomènes relèvent des partenaires scientifiques, également à disposition pour des actions ponctuelles de conseil et d'expertise. Dès le départ, l'observatoire s'est construit autour d'un SIG.

Afin d'anticiper les différents usages du SIG (archivage, visualisation, requêtes, analyses spatiales, traitements thématiques, ...), les données ont été structurées sous forme de géodatabases organisées par thématique (cf. Annexe), avec le logiciel ArcGis 10.0. Les suivis de terrain intégrés dans le SIG comprennent d’une part un état initial du littoral : typologies des plages et des contacts plage/dune, granulométrie des sables de plage, faune et flore, enjeux socio-économiques, ... Des suivis réguliers sont opérés d'autre part en vue de pouvoir analyser l'évolution du littoral dans le temps. Il s'agit notamment de profils topographiques de plage, mais aussi de suivis du contact plage/dune, du trait de côte, de la végétation ou encore des eaux souterraines. Sur proposition des partenaires scientifiques, la collectivité a acquis par ailleurs des connaissances complémentaires sur le domaine marin (bathymétrie, sédimentologie, courantologie), indispensables à la compréhension de la dynamique globale dans la cellule sédimentaire. Enfin, des suivis événementiels post-tempêtes sont également proposés (cf. Xynthia, Dirk, ...).

Outre les possibilités de visualisation des données, le SIG offre aussi de nombreuses possibilités d'exploitations des données. Ainsi, la comparaison des traits de côte digitalisés à partir des orthophotos a permis notamment via l'analyse historique d'apporter des éléments sur la dynamique d'évolution du trait de côte sur le long terme et sur le court terme (JUIGNER et al., 2014).

En complément de l'analyse historique du trait de côte, une approche des volumes de sables exportés par la tempête Xynthia (2010) a été menée. Pour faciliter l'analyse, le linéaire côtier a été découpé en 18 secteurs homogènes en croisant la distribution 


\section{XIII ${ }^{\text {èmes }}$ Journées Nationales Génie Côtier - Génie Civil \\ Dunkerque, 2-4 juillet 2014}

géographique des jeux de données utilisés (cf. ci-après). Ce découpage dédié à la question traitée respecte le degré de précision et la répartition des données utilisées (incluant photos et profils de plage).

Les reculs du trait de côte obtenus à partir des jeux d'Orthophotos (2009-2010) (JUIGNER et al., 2014) ont servi à calculer la section érodée par multiplication du recul avec les 2/3 de la hauteur moyenne de la falaise vive, hypothèse basse permettant d'approcher les différentes configurations du front dunaire avant tempête (Figure 2). L'export théorique de sable lié à l'événement Xynthia est en première approche supposé correspondre au produit de la section érodée et de la longueur du secteur.

La hauteur moyenne de falaise vive dunaire engendrée par Xynthia a été évaluée à partir des observations et mesures des falaises vives in-situ post Xynthia, en combinant (a) la hauteur moyenne du premier cordon dunaire bordier évaluée à partir de la typologie du contact plage-dune, établie pour la première fois fin juin 2010 ; (b) la hauteur de la falaise dunaire estimée à partir de l'analyse des profils de plage (et des photos correspondantes datées du 27 au 30 avril 2010) et des jeux de photos des partenaires de l’OLPM immédiatement après Xynthia (début mars 2010).

Bien qu'approximatif du fait de l'absence de mesures de terrain antérieures à Xynthia, et du mode de calcul simplifié du front dunaire, l'ordre de grandeur ainsi calculé sur le linéaire de l'observatoire du Pays de Monts $\left(80000 \mathrm{~m}^{3}\right)$ représente en un seul événement $80 \%$ du transit annuel maximum (100000 $\left.\mathrm{m}^{3} / \mathrm{an}\right)$ et 4 fois le transit minimum $(0-20000$ $\mathrm{m}^{3} / \mathrm{an}$ ) estimés par DHI \& GEOS (2008) le long de cette unité sédimentaire.

La comparaison entre les surfaces érodées lors de Xynthia et l'évolution sur le long terme du trait de côte (figure 3) montre de plus que des secteurs généralement en accrétion ou stables ont été très impactés par cette tempête. Les suivis des années suivantes ont montré une résilience nette sur la majeure partie du littoral, sauf au sud immédiat de l'hippodrome, confirmant l'impression de recul chronique du trait de côte sur ce secteur exprimée par les acteurs locaux.
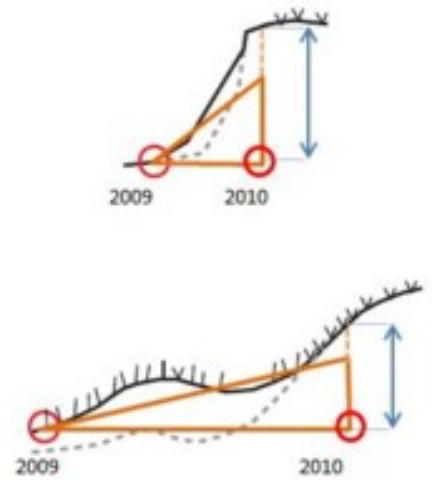
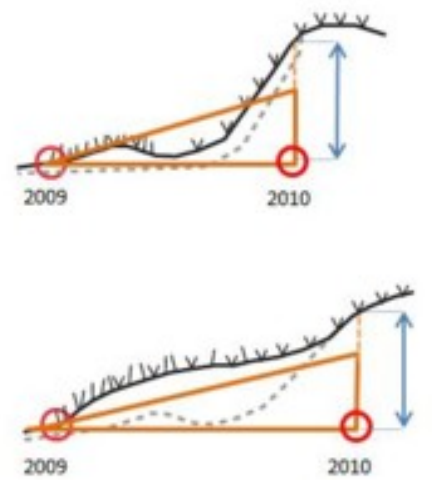

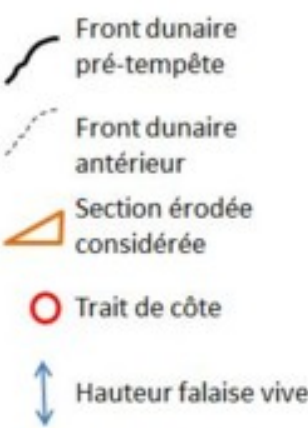

Figure 2. Représentation de la section érodée considérée dans les calculs de volume de sable exporté lors d'une tempête en fonction des configurations possibles du front dunaire avant tempête (profils stabilisés ou en accrétion schématisés ici). 


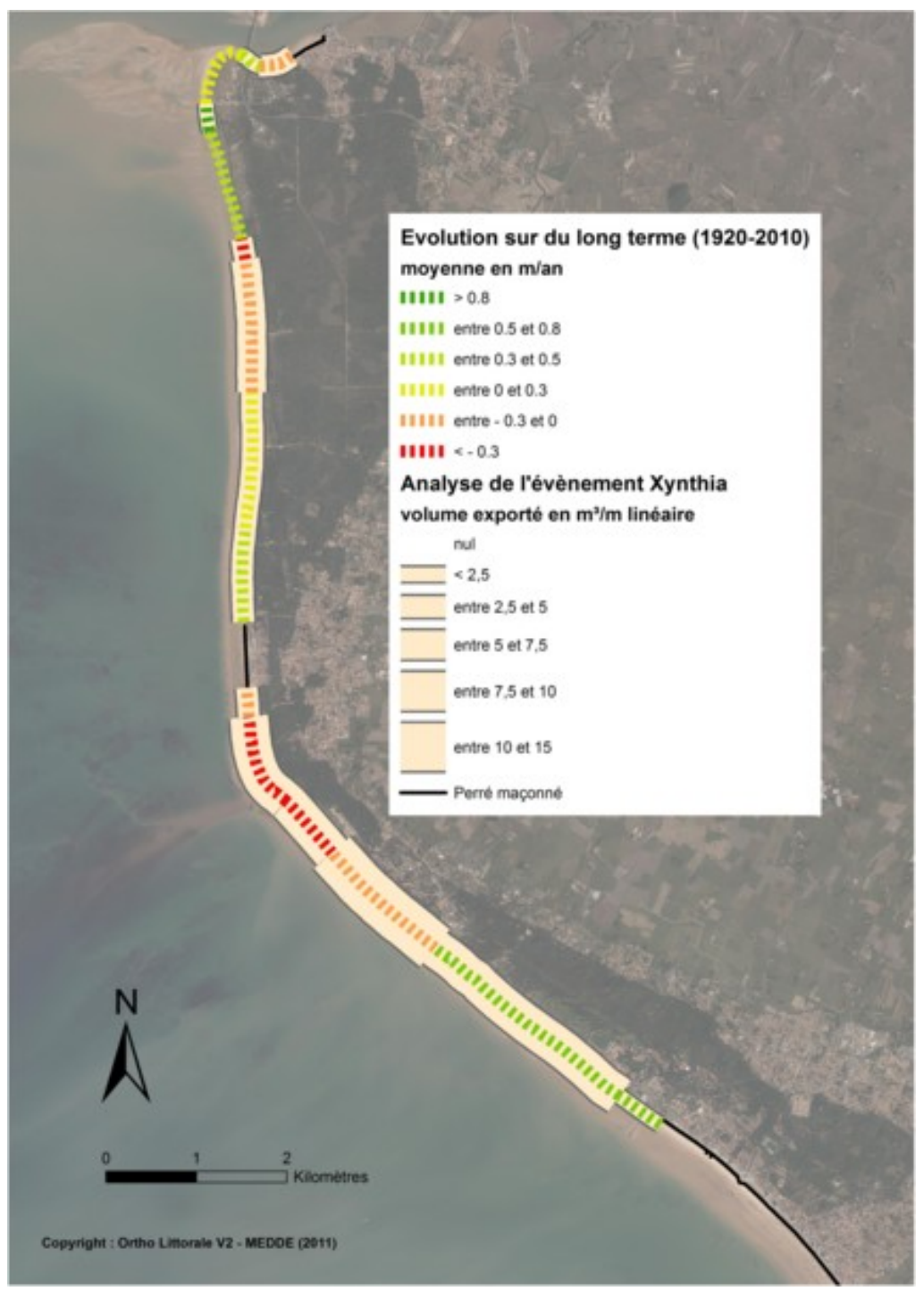

Figure 3. Comparaison de l'évolution sur le long terme du trait de côte (modifié d'après JUIGNER et al., 2014) et des volumes de sable exportés lors de la tempête Xynthia.

Les observations de mi-janvier 2014, après une série d'événements météo-marins intenses (tempête Dirk, houle Hercules) ont par ailleurs montré l'efficacité des mesures douces de protection (fils lisses) préconisées par les partenaires pour favoriser la constitution de banquettes à Agropyron. Ces dernières ont servi de premiers remparts aux assauts de la mer, et limité l'érosion de la dune. Le SIG permettra de garder la mémoire de ces observations.

Les perspectives d'exploitation des données intégrées au SIG sont plus vastes. L’outil nécessite cependant encore d’être finalisé, avec la vérification des données et des champs renseignés, la poursuite de la création des liens entre données et des métadonnées et des tests d'interrogation. 


\section{XIII ${ }^{\text {èmes }}$ Journées Nationales Génie Côtier - Génie Civil \\ Dunkerque, 2-4 juillet 2014}

\section{Conclusions}

Le bilan de mise en place l'observatoire, au bout de 5 ans, est : (a) un SIG en cours de finalisation avant livraison à la collectivité, (b) des interprétations des observations et phénomènes en jeu (évolution historique du trait de côte, volume de sable exporté lors d'un événement majeur, structure géologique du cordon dunaire, ....), et (c) des premières réponses aux questions de la collectivité ainsi que les premières applications (protection du haut de plage, sensibilisation des usagers, ...). Un rapport annuel décrit les travaux menés (LE GUERN et al., 2011 ; 2012 \& 2013).

Les connaissances acquises sur le long terme et sur le court terme, notamment sur l'évolution du trait de côte, ont permis par leur croisement de créer des cartes explicatives permettant de pondérer les conséquences sur le court terme en montrant la résilience sur un plus long terme. Il n'apparaît ainsi pas pertinent ici de mettre en place des enrochements, la mise en œuvre de solutions douces comme un fil lisse montrant son efficacité. Toutefois, sur certains secteurs en érosion chronique, un repli stratégique serait probablement à envisager à terme.

Les perspectives intègrent notamment la poursuite des suivis et interprétations, l'intégration des données en mer et la modélisation de la dynamique sédimentaire, ainsi que l'approfondissement des recommandations à la collectivité par rapport à la gestion durable du littoral. Il s’agit aussi pour la collectivité de s'approprier le SIG et de le faire perdurer.

\section{Annexe. Structure du SIG de l’observatoire du littoral du Pays de Monts}

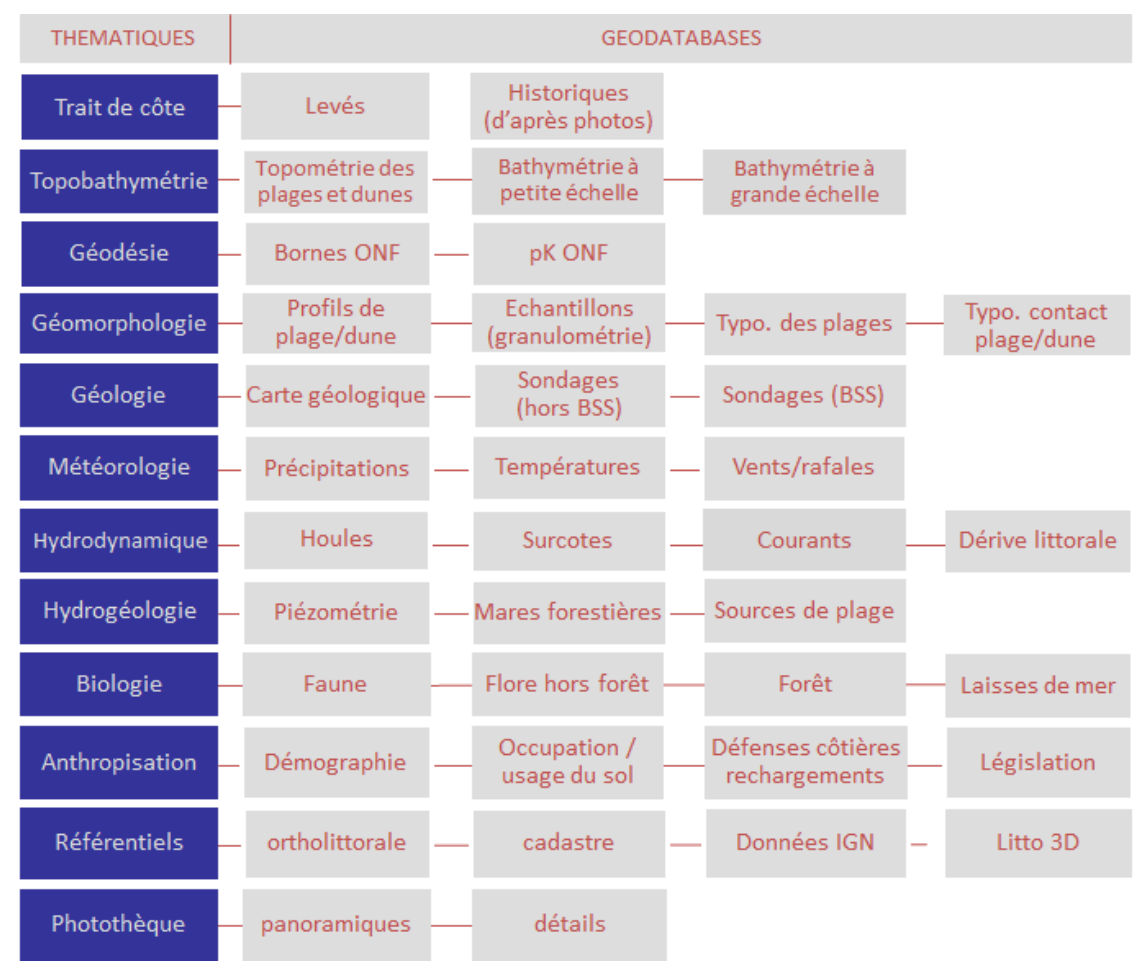


Thème 6 - Gestion durable des zones littorales et estuariennes

\section{Références bibliographiques}

BULTEAU T., GARCIN M., OLIVEROS C., LENOTRE N. (2011). Synthèse des travaux menés sur l'observation de l'évolution du trait de côte. Rapport BRGM/RP59396-FR, $156 \mathrm{p}$.

DHI, GEOS (2008). Etude détaillée de la cellule $n^{\circ} 3$ : du Goulet de Fromentine jusqu’à la pointe de Grosse Terre à Saint Gilles Croix de Vie, 74 p.

IFEN (2007). Analyse statistique et cartographie de l'érosion marine, IFEN, Les dossiers, $\mathrm{n}^{\circ} 6,37 \mathrm{p}$.

JUIGNER M., ROBIN M., FATTAL P., MAANAN M., LE GUERN C., GOUGUET L., BAUDOUIN V., DEBAINE F. (2014). Cinématique d'un trait de côte sableux en Vendée entre 1920 et 2010, In "L'homme et la dynamique littorale : maîtrise ou adaptation", sous la coordination de Y. Lageat, Y. Battiau-Queney et M.-C.Prat, Dynamiques Environnementales, numéro 30.

LE GUERN C., SUAUD S., LEGRAS L., FATTAL P., GOUGUET L., ROBIN M., BAUDOUIN V., MALLET C. AVEC LA COLLABORATION DE MAANAN M., RAMON N., DEBAINE F., RENAUL R., JAOUEN J., CONIL P., BOUCHET F., FAVENNEC J. (2011). Observatoire du littoral des Pays de Monts - Synthèse des réalisations 2010, Rapport BRGM RP-59538-FR, 211 p.

LE GUERN C., FATTAL P., GOUGUET L., BAUDOUIN V., ROBIN M., MAANAN M., DEBAINE F., JAOUEN J., RENAULT R., JUIGNER M., DURAND A., COQUIN J. avec la collaboration de P. CONIL et F. BOUCHET (2012). Observatoire du littoral des Pays de Monts - Rapport d'avancement 2011, Rapport BRGM RP-61098-FR, 88 p. LE GUERN C., JUIGNER M., BAUDOUIN V., ROBIN M., FATTAL P., ROLLIER C., MARIE D., MAANAN M. avec la collaboration DE P. CONIL, F. DEBAINE, L. GOUGUET et F. BOUCHET. (2013). Observatoire du littoral des Pays de Monts Synthèse 2012 des réalisations des partenaires scientifiques (IGARUN, ONF, BRGM), Rapport BRGM/RP-62937-FR, 95 p.

MUGICA J., MALLET C., AUBIE S. avec la collaboration de HOAREAU A., PIERSON J. (2009). Bilan des données collectées en 2007-2008 par l'Observatoire de la Côte Aquitaine. BRGM/RP-57655-FR, 51 p.

MALLET C., PREVOTEAUX B., MUGICA J., GARNIER C., HOAREAU A., AYACHE B. (2013). Observatoire de la côte aquitaine - Bilan des activités réalisées dans le cadre de la convention 2011-2012 - BRGM/RP-62591-FR, 64 p.

PRAT M-C (2008). Atelier EUCC-France - St-Jean-de-Monts et St-Hilaire-de-Riez, Rapport EUCC. p.1-4.

THINON I., BAUDOUIN V., PAQUET F., CONIL P., BECHENNEC F., LE BAYON B. (2013). Cartographie géologique harmonisée du littoral des Pays-de-la-Loire. Rapport BRGM/RP-62383-FR, 79 p. 